\title{
Desplazamiento forzado y adaptación al contexto de destino: el caso de Barranquilla
}

\author{
Ignacio Ramos Vidal*
}

Perfiles Latinoamericanos, 26(51)

2018 | pp. 301-328

DOI: $10.18504 / \mathrm{pl} 2651-012-2018$

\begin{abstract}
Resumen
En este artículo se analizan los efectos que el desplazamiento puede generar en $a$ ) la estructura de la red de apoyo social de los desplazados, $b$ ) en el empleo y $c$ ) en variables de implicación comunitaria. Se trata de un estudio comparativo en el que se entrevistó a 62 residentes en dos zonas de bajos recursos en Barranquilla, Colombia, de los que la mitad son desplazados. Entre los hallazgos se observó que el análisis de redes egocéntricas evidencia que las personas que trabajan tienen redes más diversificadas geográficamente; que los entrevistados poseen redes densas con alto grado de solapamiento, y que el tiempo de residencia es un factor clave que facilita la identificación con la comunidad y la participación en las iniciativas de desarrollo de esta. Mientras que con el análisis de conglomerados se identificaron dos grupos diferenciados por su nivel de ingreso, por el tiempo de residencia en la comunidad de destino y por el número de familiares con los que convive.
\end{abstract}

\begin{abstract}
In this article we analyze the impact that displacement could generate on: $a$ ) the displaced' social support network structure; $b$ ) employment, and $c$ ) community involvement variables. A comparative study in which are interviewed 62 people living in two low-income areas in Barranquilla city (Colombia) is developed. Half of respondents are displaced. Egocentric network analysis evidenced that people who have a job presents networks geographically diversified. The population interviewed has dense networks in which there is a high overlapping degree. Regarding community involvement indicators, is observed that time of residence is a key factor facilitating the process of identification with the community, and the involvement on participatory activities. Cluster analysis is used to identify two different groups according to 1) the income level, 2) the time who have resided in the destination community and 3) the number of family members with whom they live.
\end{abstract}

Palabras clave: cohesión social, Colombia, desplazamiento forzado, empleo, participación, redes personales, sentido de comunidad.

Keywords: Social cohesion, Colombia, forced displacement, employment, engagement, personal networks, sense of community.

* Doctor en Psicología Social por la Universidad de Sevilla. Profesor del Departamento de Psicología Social (Universidad de Sevilla) y de la Escuela de Ciencias Sociales y Humanas (Universidad Pontificia Bolivariana) | iramos5@us.es; ignacio.ramosv@upb.edu.co 


\section{Introducción}

E

n este artículo abordamos el problema del desplazamiento desde diferentes perspectivas. La primera parte del texto sigue una secuencia lógica en la que (a) en primer lugar presentamos a grandes rasgos el fenómeno del desplazamiento en Latinoamérica, para posteriormente (b) centrarnos en el caso particular de Colombia, y a continuación (c) describimos la región del Caribe colombiano, que es el contexto específico en el que se enmarca la investigación. En la introducción dedicamos una sección a las consecuencias a distintos niveles que son producto del desplazamiento. En el siguiente apartado presentamos los objetivos y el diseño de investigación, mientras que en la sección de resultados se muestran los principales hallazgos. Finalmente, en el apartado de conclusiones discutimos las implicaciones que los resultados obtenidos pueden tener para mejorar la calidad de vida de desplazados y refugiados, y la cohesión social de las comunidades que reciben población desplazada.

Quienes inician un proceso de movilidad geográfica dentro de un mismo país a consecuencia de conflictos armados, de sufrir episodios de violencia o de ser perseguidos por motivos políticos son considerados desplazados internos. Según datos del Alto Comisionado de las Naciones Unidas para los Refugiados (ACNUR), en el mundo hay en torno a 33.3 millones de desplazados internos y se han registrado más de un millón de peticiones de asilo político. Estos datos evidencian la magnitud de un fenómeno que para ser abordado de manera integral requiere de un sólido marco legal a nivel internacional para defender los derechos fundamentales de este colectivo.

Los días 2 y 3 de diciembre de 2014 se celebró en Brasilia la conmemoración del trigésimo aniversario de la Declaración de Cartagena sobre los Refugiados (Cartagena+30). La firma de este acuerdo en 1984 supuso un hito al crear un marco regulador que sirvió de base a los Estados que lo ratificaron y se comprometieron a adoptar determinadas directrices legislativas en materia de protección y promoción de los derechos de refugiados y desplazados. Su cumplimiento no es vinculante, sin embargo, en la práctica, este documento facilitó el asentamiento de un marco legal que sirviese de referencia para los países de América Latina y el Caribe. Debemos remontarnos hasta finales de la década de 1970 para comprender los factores que le dieron origen. En aquella época diversos países de América Central se encontraban sumidos en graves conflictos armados que ocasionaron un gran volumen de población desplazada. En Nicaragua, El Salvador y Guatemala la guerra civil dejó tras de sí un número de refugiados sin precedentes en la región. Tan solo en Nicaragua el conflicto armado originó más de 250000 desplazados en zonas rurales (Ortega, 1988), en 
un país que en la época del conflicto apenas alcanzaba los seis millones de habitantes. Ejemplos como este pusieron en el punto de mira la necesidad de crear un marco regulador en materia de acogimiento y de protección de los derechos de desplazados y refugiados.

Como afirma Demant (2013: p. 135), la Declaración de Cartagena de 1984 sentó un importante precedente en la temática al señalar la importancia que el acceso a derechos económicos, sociales y culturales tales como la salud, la educación y las oportunidades de empleo y medios de vida tiene para asegurar la integración local de los refugiados. Sin embargo, en la actualidad las causas - y consecuencias - del desplazamiento presentan notables diferencias respecto a las que motivaron la creación de un marco común en materia de protección de los derechos que asisten a la población desplazada. Actualmente, la guerra civil en sí misma no es la causa esencial del desplazamiento masivo, a diferencia de lo que sucediera a finales de 1970 y durante los años ochenta. El desplazamiento en América Latina, ${ }^{1}$ por el contrario, tiene en nuestros días múltiples vertientes que reflejan diferentes problemas y desequilibrios sociales que se encuentran en la base del surgimiento de nuevos desplazados y que producen notables transformaciones en la estructura poblacional de los contextos de origen y de destino. Un claro ejemplo lo encontramos en el gran número de emigrantes mexicanos que residen en Estados Unidos (Albo \& Ordaz, 2011). Una cantidad considerable de estas personas son desplazadas en la práctica por huir de situaciones de violencia y por la presión que los grupos criminales ejercen sobre el territorio para controlar las rutas del tráfico de estupefacientes. Por ejemplo, en México, desde 2009, se han registrado 248418 casos de desplazamiento forzado debido al régimen de violencia impuesto por los cárteles de la droga (Rubio, 2014). Aunque, según este mismo estudio, tal número debe considerarse como una aproximación conservadora puesto que estimaciones del Instituto Nacional de Estadística y Geografía (INEGI) ${ }^{2}$ cifran en 1.6 millones los desplazados en ese mismo periodo. Para ilustrar la inherente complejidad de este fenómeno, cabe mencionar que este mismo país (México), además de ser un destacado emisor de población, al mismo tiempo es un país receptor (y de tránsito) de desplazados y refugiados provenientes de Centroamérica y de países del Cono Sur.

Otro elemento que agudiza el problema del desplazamiento y que genera nuevas situaciones de indefensión y vulneración de derechos humanos es el cambio que ha experimentado el perfil del desplazado. El número de desplazados menores de edad que viajan solos se incrementó de modo exponencial en

1 En la obra compilada por Susana Novick se examinan diferentes procesos de movilidad entre regiones de América Latina mostrando varias experiencias y estudios de caso.

2 http://www.inegi.org.mx

I. Ramos Vidal | Desplazamiento forzado y adaptación al contexto de destino: el caso de Barranquilla Perfiles Latinoamericanos, 26(51) Flacso México | pp. 301-328 | DoI: 10.18504/pl2651-012-2018 
los últimos años. Un ejemplo de esto es el volumen de niños procedentes de Honduras que viajan hacia Estados Unidos huyendo de situaciones de violencia y pobreza. Solo en 2014 más de catorce mil menores hondureños entraron de manera irregular en Estados Unidos. Esto es una muestra de los nuevos fenómenos que se encuentran en la base del desplazamiento en un momento en el que los efectos del estancamiento económico de la región, la proliferación de los cárteles de la droga, el incremento de las desigualdades sociales y el aumento de pandillas y maras propician un aumento en el número de personas que se ven obligadas a abandonar la comunidad de origen persiguiendo un futuro mejor, o simplemente un futuro.

Por tanto parece que, a diferencia de lo que sucediera en décadas anteriores, las causas del desplazamiento no se restringen a la guerra o a conflictos armados, sino que están surgiendo nuevos fenómenos sociales que inciden sobre el desplazamiento forzado. Entre estas nuevas situaciones destacan el crecimiento demográfico, la urbanización, la débil gobernanza, la inseguridad alimentaria y energética, la escasez de agua, los desastres naturales, el cambio climático y la crisis financiera (Demant, 2013; Guterres, 2011). A continuación abordamos el fenómeno del desplazamiento en el contexto colombiano.

El ordenamiento jurídico colombiano aborda de forma específica el problema del desplazamiento. La Ley 387 de 1997 define que el desplazado interno es toda persona que se ha visto forzada a migrar dentro del territorio nacional, abandonando su localidad de residencia y sus actividades económicas habituales debido a que su vida, su integridad física, su seguridad o sus libertades individuales han sido vulneradas o se encuentran directamente amenazadas con ocasión de cualquiera de las siguientes situaciones: (a) conflicto armado interno, (b) disturbios y tensiones, (c) violencia generalizada, (d) violaciones masivas a los derechos humanos, (e) infracciones del derecho internacional humanitario $\mathrm{u}$ otras circunstancias emanadas de las situaciones anteriores que puedan alterar o alteren drásticamente el orden público.

Colombia es el país latinoamericano que más desplazados internos genera por motivo de la violencia política; únicamente Sudán, que padeció durante varias décadas una guerra civil, la supera en el ranking de naciones con mayor número de desplazados internos (IDMC, 2011). El desplazamiento en Colombia se debe a un conflicto histórico que se ha extendido por más de cuarenta años y ha dejado importantes secuelas en la sociedad civil, afectando a la estabilidad de las instituciones. Por otro lado, la complejidad del fenómeno en el que se mezclan elementos de carácter político, económico y social, lo convierten en un proceso dinámico con múltiples alteraciones a lo largo de los últimos años (Restrepo, Spagat \& Vargas, 2003). De hecho no han faltado los intentos por resolver el conflicto mediante diálogos multilaterales (Fisas, 2010). 
La última de estas iniciativas concluyó el 24 de agosto de 2016 con un acuerdo entre la cúpula de las Fuerzas Armadas Revolucionarias de Colombia (FARC) y el gobierno colombiano en la ciudad de La Habana. ${ }^{3}$ Este acuerdo programático deberá ser ratificado en un plebiscito el 2 de octubre de 2016. Sin embargo, cabe señalar que en el conflicto colombiano - y en la venidera etapa del posconflicto- entran en juego otros actores relevantes, como grupos paramilitares vinculados al mundo del narcotráfico, el Ejército de Liberación Nacional (ELN) y facciones de las FARC que no están de acuerdo con el proceso de negociación y que pueden hacer peligrar la consecución real de la paz.

El enfrentamiento entre los grupos armados ilegales y el ejército es otro de los elementos que contribuyen de forma significativa al incremento de la población desplazada. Los paramilitares maniobran para apoderarse de lo que pueden considerarse zonas estratégicas para incrementar el control territorial, por ejemplo, para generar corredores que permiten trasladar cargamentos de drogas hacia zonas costeras. Existen regiones como la del Chocó, en la costa pacífica, en donde el poder y el control fáctico de estos grupos dificulta la presencia del ejército y ocasiona numerosos conflictos (Oslender, 2007). Las personas que residen en zonas rurales que habitan en el área de influencia de la guerrilla o de los paramilitares abandonan sus tierras ante las amenazas directas de estos grupos.

Esta situación es un drama que viven más de cinco millones de personas de todos los rangos de edad a lo largo de la geografía colombiana (IDMC, 2011). En 2011, coincidiendo con la promulgación de la Ley de Víctimas y Restitución de Tierras (Ley 1448), dictada por el Gobierno de la República de Colombia, se observó un repunte significativo de la violencia por parte de grupos ilegales. Este hecho puede interpretarse como una reacción por parte de las organizaciones delictivas para promover que los damnificados no hagan valer sus legítimos derechos sobre la tierra, instaurando una política basada en el terror para lograr sus fines.

A continuación se describen algunas de las principales consecuencias del desplazamiento. Promover la movilidad de personas o colectivos es considerada una flagrante violación de los derechos humanos por parte del derecho internacional (ASCODAs, 2004). Tanto en el contexto internacional como en el nacional, existe una gran ambigüedad en la definición legal del término, y en cuanto a los refugiados de Colombia no se ha establecido una legislación clara al respecto. Esta falta de definición se erige en un problema que dificulta la articulación de

3 El texto completo del Acuerdo final para la terminación del conflicto y la construcción de una paz estable y duradera, como se le ha denominado formalmente, se consultó el 2 de septiembre de 2016 y está disponible en http://www.elespectador.com/noticias/paz/el-texto-completo-de-los-acuerdos -firmados-habana-articulo-639605 
las acciones y políticas que deben desarrollar las instituciones para enfrentar el problema del desplazamiento.

Una de las consecuencias más notorias de tal fenómeno en Colombia es su efecto en la configuración de las zonas urbanas (Naranjo, 2004). El éxodo desde áreas rurales a las urbanas produce importantes alteraciones en la estructuración natural de ambos entornos: genera despoblación en las primeras e incrementa la presión demográfica en las segundas. Aunque hay multitud de iniciativas a nivel gubernamental y por parte de la sociedad civil para atajar la desigualdad social producto de la violencia, no es menos cierto que muchas de ellas se han mostrado ineficaces, a veces por la magnitud del fenómeno (Caicedo, 1997) y en otras por falta de voluntad política y consenso institucional (Estrada, 2010).

Aunque en este artículo nos enfocamos en el proceso de movilidad que experimentan los desplazados que se trasladan desde regiones del interior hacia zonas costeras, también se han documentado procesos de movilidad intraurbana que igualmente producen importantes cambios en la vida de la población desplazada. Desde esta perspectiva, Sánchez (2008) documenta este fenómeno en Medellín. El trabajo desarrollado por la autora constata que los procesos de movilidad que tienen lugar dentro de la misma ciudad, al igual que los procesos de movilidad interdepartamentales, muestran una notable capacidad para producir situaciones de desarraigo, debilitamiento de lazos sociales y de afectar la calidad de vida.

Los efectos del desplazamiento se sienten de forma distinta en las diferentes regiones del país. Tradicionalmente el departamento del Atlántico es uno de los que más desplazados recibe. Según Hernández (1999: p. 3), dicha entidad recibe el $21.7 \%$ de los desplazados internos del país. Los asentamientos de población desplazada se sitúan en las cabeceras municipales, donde es frecuente que una amplia proporción de los habitantes proceda de diferentes regiones (Flores $\&$ Crawford, 2010). El estudio que hemos realizado se desarrolla en la ciudad de Barranquilla, en el departamento del Atlántico. Por este motivo centraremos la descripción del contexto de investigación en esta zona del Caribe colombiano.

La población que experimenta violencia, o que debe huir ante la amenaza de grupos armados, busca recursos que le permitan sobrevivir en el nuevo contexto de asentamiento. Este proceso de ajuste implica que la persona desplazada ubique su residencia en zonas de bajos recursos que habitualmente se sitúan en la periferia de las ciudades. La mayor parte de los desplazados se asientan en zonas de estratos 1 y $2 .{ }^{4}$ Estas poblaciones se caracterizan por presentar múlti-

4 En Colombia, las zonas metropolitanas se diferencian en función de los estratos sociales. Para los detalles de la distribución por estratos en Barranquilla, véase http://roble.barranquilla.gov.co:8888/images/ Valorizacion2/Normatividad/7_Estudio_Socioeconomico.pdf 
ples problemas económicos y sociales que derivan en notables deficiencias de los servicios socio-sanitarios y educativos (Ramos-Vidal, Rodríguez, Palacio, \& Maya-Jariego, 2013). ${ }^{5}$ Por otro lado, las infraestructuras dedicadas a la prestación de servicios son precarias generalmente, al igual que las instalaciones sanitarias como las de abastecimiento de aguas. Por este motivo, a los problemas sociales ocasionados por la falta de recursos de asistencia social, hay que añadir los sanitarios que afectan a estas poblaciones y que son fruto del crecimiento demográfico no planificado.

Uno de los elementos propios de Barranquilla es que, dentro de sus límites geográficos, se encuentran varios distritos como Soledad, Los Ángeles y Puerto Colombia en los que reside una vasta proporción de desplazados (Flores $\&$ Crawford, 2010). Esta concentración puede llegar a producir conflictos entre la población local y la desplazada de reciente ingreso. Palacio \& Sabatier (2002) demostraron que una de las consecuencias más notables del desplazamiento es la segregación que se genera en las comunidades que lo reciben en grandes contingentes. Esto se produce debido a que los habitantes locales perciben a los desplazados como potenciales competidores por los recursos. Esta polarización puede resultar en conflictos sociales si no se gestiona adecuadamente.

La población desplazada suele encontrar dificultades para salir adelante en el contexto de destino. El debilitamiento o ruptura de los vínculos con la comunidad de origen y la falta de contactos en el destino dificulta el acceso a recursos. Por tal causa es frecuente que quede expuesta a situaciones de exclusión social y deba recurrir a la mendicidad, la prostitución y la delincuencia, como alternativas para sobrevivir y obtener ingresos para mantener a sus familias (Caicedo, 1997). La precariedad laboral y el desempleo son factores que distinguen a la población desplazada. Muchas de estas personas provienen de zonas rurales en las que se dedicaban al sector agrícola. En las ciudades a donde llegan, como Barranquilla, la actividad que más empleo produce es la industria y en menor medida los servicios, por lo que muchos desplazados no consiguen un trabajo relacionado con la actividad que realizaban en su lugar de origen. Por lo general esta población se ocupa en trabajos precarios poco cualificados del sector de servicios. Otra opción frecuente es trabajar en microempleos, por lo común subcontratando puestos de venta ambulante. La mayor parte de los desplazados se incorpora a la economía informal o en los servicios y están mal remunerados (Cruz, 2008).

Debemos considerar otros efectos que son consecuencia directa del desplazamiento y que quizá no resulten tan visibles como lo arriba expuesto. Son efectos que se producen a nivel (a) individual, (b) familiar, (c) comunitario y

5 El citado informe se encuentra disponible bajo petición al autor. 
(d) macrosocial. Enseguida se resumen los efectos que el desplazamiento produce en cada uno de ellos.

A nivel personal, la gente que abandona su comunidad experimenta una reducción de sus fuentes de apoyo social. La distancia y la falta de contacto hacen que los lazos sociales se debiliten y que la persona deje de disponer de sus fuentes de apoyo material, afectivo e informativo, entre otros. Esta pérdida tiene importantes consecuencia psicosociales. Las personas que disponen de redes de apoyo amplias y heterogéneas tienen menos probabilidades de padecer estrés y depresión (Taylor \& Brown, 1988). Los estudios acerca del contexto relacional de la población inmigrante documentan el rol moderador del apoyo social en el bienestar de los inmigrantes (Martínez, García \& Maya, 2001). El análisis de las redes personales o egocéntricas de la población migrante permite evaluar desde un enfoque estructural la adaptación psicosocial en el contexto de destino (De Miguel \& Tranmer, 2010; Lubbers, Molina, Lerner, Brandes, Ávila \& McCarty, 2010).

A nivel familiar el desplazamiento produce una fragmentación de la estructura de la unidad familiar. No obstante, cuando emigra toda la familia el efecto no es tan significativo puesto que permanece la unión y aunque el impacto del cambio de residencia se mantenga, será menor puesto que las principales fuentes de apoyo siguen disponibles. Pero en lo general las familias no migran en bloque, el desplazamiento se produce de forma escalonada, lo que provoca cierto grado de descomposición en la estructura familiar (Forero, 2003). Un efecto más es la pérdida de referentes por el alejamiento de los miembros de la familia. Este fenómeno conduce a la pérdida de identidad familiar y a la anomía. Además, la salida de un miembro de la unidad familiar tiene efectos en la economía doméstica, en particular cuando quien se traslada es el cabeza de familia.

La esfera que más se ve afectada por el desplazamiento forzado después de la familia es el contexto comunitario. Por toda la geografía colombiana, en particular en el departamento del Chocó y del Valle del Cauca, es posible encontrar localidades que han visto mermar su población por el éxodo masivo a las grandes ciudades. Con esto el tejido social queda gravemente dañado. Muchas de estas localidades están habitadas por pocas familias que viven de la agricultura; al abandonar sus tierras, la comunidad se reduce y a veces directamente desaparece. Las comunidades de destino, por su parte, padecen los efectos del desplazamiento. En los asentamientos de las cabeceras municipales que más población acogen crece la segregación social y se producen problemas de convivencia. Esta misma polarización produce debilitamientos de la cohesión social, lo que afecta negativamente en la dinámica social.

Por último, a nivel macrosocial se observan cambios de largo alcance que afectan a toda la población del país, no solo a desplazados y a municipios receptores y emisores. Builes, Arias \& Minayo (2008: p. 1652) afirman que el 
fenómeno del desplazamiento en Colombia presenta tres elementos que lo diferencian de otros países: a) la continuidad histórica del proceso, b) la multipolaridad del conflicto y c) la falta de identidad de la población desplazada, que es una consecuencia de los dos primeros factores mencionados. En la mayor parte de países con desplazamiento poblacional, este se debe a coyunturas específicas como desastres medioambientales, conflictos bélicos o brotes puntuales de violencia. Sin embargo, en Colombia el conflicto es histórico y continuado durante más de cuarenta años, se ha convertido en una característica endémica del país. La segunda peculiaridad se debe a la participación en el conflicto de múltiples actores y organizaciones delictivas como las FARC, el FLN, las organizaciones del narcotráfico y las distintas facciones paramilitares. Este aspecto hace aún más difícil una salida negociada al conflicto dada la gran cantidad de actores e intereses enfrentados que entran en juego. El tercer y último factor es la falta de identidad de la población desplazada. Esto se debe fundamentalmente a que se trata de un colectivo heterogéneo con pocos rasgos en común y que proviene de distintas regiones, lo que dificulta el establecimiento de un sentido de comunidad (McMillan \& Chavis, 1986). Todo esto produce fuertes consecuencias sobre el nivel de confianza de la sociedad colombiana e incide negativamente en la cohesión social.

Los niveles afectados por el desplazamiento forzado han sido identificados. Si bien es preciso enfatizar la interdependencia entre los diferentes niveles examinados. Así, los efectos individuales trascienden inmediatamente a la esfera familiar, luego al ámbito comunitario y finalmente sobre la sociedad en su conjunto. Pero este proceso no solo se produce desde el nivel microsocial hasta el macrosocial, sino que es también permeable entre los diferentes niveles lo que genera un impacto de los niveles superiores hacia los inferiores, y viceversa. Una vez descritas las características del contexto de estudio y los problemas relativos al desplazamiento, en la siguiente sección se presentan los objetivos y el diseño de investigación.

\section{Objetivos}

El propósito de este estudio consiste en conocer los efectos del desplazamiento en la población que experimenta esa situación y en las comunidades afectadas por tal fenómeno. Para ello se analizan aquí variables de tipo 1) relacional, 2) laboral y 3) de implicación comunitaria. Se presentan brevemente las áreas que se han evaluado.

A nivel relacional se ha evaluado la estructura de las redes de apoyo social de la población desplazada (McCarty, 2002). El análisis estructural permite conocer 
los efectos del desplazamiento para el entorno relacional de las personas. Existen configuraciones estructurales que facilitan la adaptación en el nuevo contexto y que incluso generan capital social y oportunidades de desarrollo (Ramos-Vidal, 2015). La literatura sobre apoyo social destaca que quienes disponen de una sólida red que les brinda distintos recursos sociales, son capaces de afrontar con más garantía las adversidades y padecen menos estrés y enfermedades de salud mental (Cohen $\&$ Wills, 1985). En este sentido, la red social es un recurso protector. Sin embargo, otros estudios ponen de manifiesto que es necesario que además de un alto grado de cohesión interna, también deben haber relaciones con grupos externos, puesto que estos vínculos abren el acceso a recursos e información para ascender en el escalafón social (Granovetter, 1973). Por este motivo en particular, la población desplazada que disponga de este tipo de vínculo que la literatura denomina de tipo bridging, puede ser decisivo para superar situaciones de exclusión social.

A nivel profesional se han examinado las características del empleo en el que se desempeña la población objeto de estudio. Otro elemento clave para conocer el nivel de adaptación de los desplazados en el contexto de destino es el grado de empleabilidad y las opciones de ocupación. Para ello se han analizado variables relativas al desarrollo de actividad remunerada, al tipo de trabajo y al modo en que un desplazado se vincula a las organizaciones en las que trabaja. Tener un trabajo estable constituye un indicador del nivel de adaptación social; es información que hace posible comprender el grado de ajuste de la población en el nuevo entorno comunitario. También se examina el nivel de educativo de la población.

Por último, a nivel comunitario, se decidió estudiar el grado de participación de la población en la comunidad de destino. La implicación en actividades de promoción comunitaria es un factor esencial para garantizar el bienestar de la población (Kloos et al., 2011; Wandersman, 2009). Los miembros de la comunidad tienden a participar en la medida en que perciben que forman parte de una colectividad superior. Precisamente este es otro de los constructos evaluados al que se ha denominado sentido de comunidad (SC). Este proceso examina qué tanto los individuos se miran a sí mismos como miembros de una comunidad de carácter geográfico y/o relacional. Según McMillan \& Chavis (1986), el SC consta de cuatro dimensiones: $a$ ) la pertenencia, b) la influencia, c) la satisfacción de necesidades y d) la conexión emocional compartida. La pertenencia analiza el grado en el que los individuos advierten que forman parte de una colectividad mayor. La influencia mide si los individuos al formar parte de la comunidad perciben que pueden influir y ser influidos por la colectividad a 
la que pertenecen. La satisfacción de necesidades examina la medida en que, al participar en la comunidad, los miembros toman conciencia de que pueden satisfacer sus propias necesidades, al mismo tiempo que contribuyen a satisfacer las del resto de los miembros del grupo. Finalmente, la conexión emocional compartida evalúa la existencia de un código de valores compartido que dota de significado al grupo. El análisis de este constructo es de vital importancia para comprender la interacción entre el individuo y la comunidad y el funcionamiento interno de esta. Además, diferentes autores enfatizan el papel modulador del SC como facilitador de la participación comunitaria (Chavis \& Wandersman, 1990; Ramos-Vidal, Holgado \& Maya-Jariego, 2014; Ramos-Vidal \& MayaJariego, 2014). Presentados los objetivos del estudio, en la siguiente sección se describe el diseño de la investigación mostrando las características de los participantes, de las variables y de los instrumentos empleados.

\section{Método}

El estudio desarrollado aquí es transversal, comparativo y de naturaleza exploratoria. Se llevaron a cabo 62 entrevistas con residentes de dos comunidades de bajos recursos de Barranquilla en el departamento del Atlántico, Colombia. De los 62 encuestados, 30 se encuentran en situación de desplazamiento y los 32 restantes no. El principal motivo del desplazamiento ha sido la violencia, aunque algunos casos fueron consecuencia de las inundaciones que se produjeron en la región del Atlántico en diciembre de 2010. De la población desplazada se tuvo que eliminar un caso en el que no se completó el cuestionario. El tiempo medio de desplazamiento desde la salida de la comunidad de origen fue de 8.9 años (DT $=9.03$ ).

En lo referente al protocolo para la obtención de datos, las entrevistas fueron aplicadas por un equipo de encuestadores adiestrados por los responsables del estudio. El trabajo de campo se realizó entre marzo y junio de 2013. Los participantes firmaron un consentimiento informado, se les indicó que se preservaría su anonimato en todo momento y se haría un uso agregado de la información. Los encuestadores también firmaron un compromiso de confidencialidad antes de la instrucción.

\section{Descripción de los participantes}

Dado que el objetivo es analizar los efectos del desplazamiento en el proceso de adaptación en la comunidad de destino, se realizó un estudio comparativo 
entre población desplazada y no desplazada. En cuanto a la distribución por sexo, participaron mujeres en su mayoría $(\mathrm{n}=55 ; 88.7 \%)$ en comparación con los varones $(\mathrm{n}=11 ; 11.3 \%)$. Esta desigualdad se debe a que las personas fueron entrevistadas en fundaciones de atención a población infantil en horario matinal, por lo que la mayor proporción de personas que acuden a este tipo de centros - a dejar o recoger a los hijos- son mujeres. En la distribución por sexo entre la población desplazada se conservó la mayoría femenina $(\mathrm{n}=25 ; 86.2 \%)$ en relación a los hombres $(\mathrm{n}=4 ; 13.8 \%)$; misma tendencia que se observó en el grupo de no desplazados (29 mujeres, 90.6\%; 3 varones; 9.4\%). En el cuadro 1 se resumen las características sociodemográficas de la población evaluada.

Cuadro 1. Media y desviación tipo de la edad, nivel de ingreso e integrantes de la familia

\begin{tabular}{lcccc}
\hline & \multicolumn{2}{c}{ Desplazados $(n=29)$} & \multicolumn{2}{c}{ No Desplazado $(n=32)$} \\
\cline { 2 - 5 } Variables & Media & $D T^{*}$ & Media & $D T$ \\
\hline Edad & 33.5 & 10.9 & 33.4 & 13.9 \\
Nivel de ingreso mensual (US\$) & 274.47 & 142.71 & 289.59 & 190.65 \\
$N^{\circ}$ de miembros en la unidad familiar & 6.1 & 6.1 & 4.6 & 2.8 \\
Ingreso per cápita mensual & 45.7 & 23.39 & 62.95 & 67.85 \\
\hline
\end{tabular}

*DT: Desviación tipo.

Fuente: Elaboración propia.

Se puede apreciar que las características de ambas poblaciones por edad, sexo y en función del nivel de ingreso es sustancialmente similar. Un dato destacado es que ninguna de las dos poblaciones alcanza el salario mínimo interprofesional fijado por el gobierno para el año 2013 (equivalente a 332.76 dólares mensuales). Si se tiene en cuenta que los ingresos a los que alude la tabla 1 son los recursos de la unidad familiar completa, al dividir los ingresos mensuales por el número de miembros, resulta que cada integrante de la familia vive con 1.5 dólares diarios. ${ }^{6}$ Entre la población desplazada esta cifra es ligeramente superior, sin embargo, en ambos casos los ingresos están cercanos a los niveles de pobreza extrema que establecen organismos internacionales como el Programa de las Naciones Unidas para el Desarrollo (PNUD).

El nivel educativo de la población es un indicador que incide en el nivel de bienestar subjetivo (Diener, Sandvik, Seidlitz \& Diener, 1993), en la esperanza de vida (Lleras-Muney, 2005) y en el acceso a puestos de trabajo cualificados bien remunerados (Galor \& Zeira, 1993). En el cuadro 2 se presenta la información relativa al nivel de educación diferenciando entre las dos poblaciones analizadas.

6 La Comisión Económica para América Latina y el Caribe (CEPAL) cifró el umbral de la pobreza en vivir por debajo de 1.25 dólares diarios. 
Cuadro 2. Nivel educativo de la población encuestada

\begin{tabular}{lcccccc}
\hline \multirow{2}{*}{$\begin{array}{l}\text { Grado } \\
\text { educativo }\end{array}$} & \multicolumn{2}{c}{ Desplazados $(n=29)$} & \multicolumn{2}{c}{ No Desplazados $(n=32)$} & \multicolumn{3}{c}{ Total } \\
\cline { 2 - 6 } Sin estudios & $n$ & $\%$ & $n$ & $\%$ & $n$ & $\%$ \\
Primaria & 1 & 3.4 & 1 & 3.1 & 2 & 3.2 \\
Secundaria & 16 & 55.2 & 12 & 37.5 & 28 & 46.8 \\
Universitaria & 11 & 37.9 & 15 & 46.9 & 26 & 41.9 \\
Perdidos & 1 & 3.4 & 2 & 6.3 & 2 & 3.2 \\
Total & 1 & 3.4 & 2 & 6.3 & 3 & 4.8 \\
\hline
\end{tabular}

Fuente: Elaboración propia.

Los datos del cuadro 2 indican que se trata de población en la que predomina el nivel educativo medio, y que es poco frecuente que los participantes carezcan por completo de estudios $(3.2 \%)$ o que tengan educación universitaria (3.2\%). Así, la mayor parte de los entrevistados (88.7\%) cursó estudios de nivel intermedio. En el cuadro 3 se ofrece información sobre el desempeńo de actividades profesionales y el tipo de actividad y vinculación de ambos colectivos.

Cuadro 3. Desarrollo de actividades profesionales

\begin{tabular}{lcccccc}
\hline \multirow{2}{*}{$\begin{array}{l}\text { Trabajo } \\
\text { remsunerado }\end{array}$} & \multicolumn{2}{c}{ Desplazados $(n=29)$} & \multicolumn{2}{c}{ No Desplazados $(n=32)$} & \multicolumn{2}{c}{ Total } \\
\cline { 2 - 7 } & $N^{\circ}$ de casos & $\%$ & $N^{\circ}$ de casos & $\%$ & $N^{\circ}$ de casos & $\%$ \\
\hline Sí & 14 & 44.8 & 12 & 40.6 & 26 & 41.9 \\
No & 15 & 55.2 & 18 & 53.1 & 34 & 54.8 \\
Perdidos & -- & -- & 2 & 6.3 & 2 & 3.2 \\
Total & 29 & 100 & 32 & 100 & 62 & 100 \\
\hline
\end{tabular}

Fuente: Elaboración propia.

Los datos reflejan que en los dos grupos de encuestados hay tendencias similares en la ocupación. En torno a la mitad de los entrevistados manifestó no realizar actividad profesional remunerada, mientras que la otra mitad indicó recibir contraprestación económica por el desarrollo de actividad laboral. En la mayor parte de casos en los que la mujer indicó no realizar actividad profesional fuera del hogar, al mismo tiempo señaló que era ella quien asumía las tareas domésticas, pero que no percibía recompensas por este trabajo. Por tal motivo es necesaria la cautela al interpretar este dato. De todas formas y dado que el trabajo doméstico es fundamental para el desarrollo de la actividad productiva remunerada y diversos autores (Portes, 1995) seńalan la importancia de las actividades informales para que exista la economía global, es evidente que el desempleo es un rasgo inherente de la población estudiada. 
Cuadro 4. Actividad que desarrolla la población que trabaja

\begin{tabular}{lccc}
\hline Sector & Desplazados $(n=14)$ & No Desplazados $(n=12)$ & Total \\
\hline Doméstico & 8 & 4 & 12 \\
Servicios & 5 & 7 & 12 \\
Agrícola & 1 & 1 & 2 \\
Total & 14 & 12 & 26
\end{tabular}

Fuente: Elaboración propia.

Los participantes que realizan actividad remunerada se distribuyen equitativamente entre el empleo en el servicio doméstico y en el sector de servicios. La dedicación al sector agrícola es residual. Esto se explica porque la actividad económica en Barranquilla se da sobre todo en los servicios. Y en lo referente al tipo de vinculación de la población desplazada que realiza actividad remunerada, solo el $10.3 \%$ manifestó una relación contractual con el empresario mientras que, entre los no desplazados, el $21.9 \%$ de los entrevistados que afirmó realizar actividad laboral remunerada, señaló disponer de contrato profesional. A pesar del reducido número de casos disponibles, que los desplazados laboren en trabajos precarios en mayor proporción a los no desplazados parece un indicador ilustrativo de la situación del colectivo. A continuación se exponen las características de los instrumentos utilizados para analizar el contexto relacional de los entrevistados y su vínculo con la comunidad.

\section{Instrumentos}

Para estudiar las redes de apoyo social se analizó la estructura de la red egocéntrica de los entrevistados. Para que la persona identificase a los integrantes de su red se utilizó el generador de nombres assis (Arizona Social Support Interview Squedule) propuesto por Barrera, Sandler \& Ramsay (1981). El instrumento incluye seis tipos de preguntas referentes a diferentes clases de apoyo (material, informativo, afectivo, etc.) que deben inspirar al entrevistado a listar los contactos de su red personal. Además, este instrumento recoge el tipo de relación que vincula a "ego" (la persona entrevistada) con las personas que son parte de su red ("alter"). Asimismo se preguntó por las características (atributos) de los actores que conforman esa red. Se solicitó información sobre su vínculo con el entrevistado, la procedencia de los alter y por la multiplicidad de apoyos que proporcionan a ego. Una vez obtenidas las redes, se calcularon las medidas de cohesión con el software Ucinet 6.3 (Borgatti, Everett \& Freeman, 2002).

Se eligieron diversas medidas que, según McCarty (2002), permiten comparar la estructura de las redes personales. Los indicadores estructurales utilizados 
fueron la densidad, que señala la proporción de contactos que tiene lugar en una red en relación al total de vínculos posibles (Harary, 1969); la centralización de la intermediación, que muestra el grado en que las relaciones de la red completa tienen capacidad para interconectar a los diferentes subgrupos que la integran. ${ }^{7}$ Y el número cliques, que señala los subgrafos de al menos tres actores en los que todos los miembros están conectados entre sí (Luce \& Perry, 1949). Una vez calculados los indicadores de cohesión se creó una base de datos con el software sPss (20.0).

Para evaluar la participación comunitaria se empleó la escala propuesta por Speer \& Peterson (2000). Es un instrumento que consta de cinco ítems que indagan sobre el nivel de implicación en acciones participativas en diferentes instituciones de la comunidad a través de distintas vías: los participantes debían indicar en una escala tipo likert de cinco puntos que oscilaban desde "nunca participó" a "todas la semanas". La escala de participación comunitaria cuenta con óptimas propiedades psicométricas. En este estudio se ha reportado un Alpha $=.86$, lo que la sitúa dentro de los parámetros de confiabilidad (DeVellis, 1991).

El SC ha sido evaluado con la escala SCI-II (Sense of Community Index-II) propuesta por Chavis, Lee \& Acosta (2008). Este instrumento consta de 24 ítems que los participantes puntúan en una escala tipo likert de 1 a 4 en función del grado de acuerdo que manifestaban ante diferentes afirmaciones (p. ej., "creo que mi comunidad es un buen lugar en el que vivir" o "las personas de esta comunidad tienen necesidades, prioridades y metas similares"). La escala goza de propiedades psicométricas óptimas en la evaluación de las cuatro dimensiones. Hemos reportado una fiabilidad total de la escala $\alpha=.89$. Así mismo, el índice de fiabilidad de los cuatro factores de la escala se sitúa entre el .80 y el .90 , mostrando excelentes propiedades psicométricas. En el siguiente apartado se detallan los principales resultados de la investigación.

\section{Resultados}

Continuando con la misma secuencia que hemos seguido a lo largo del texto, presentamos los principales hallazgos relativos a la estructura de las redes personales y a los efectos del desplazamiento sobre el empleo y sobre los indicadores

7 McCarty (2002) señala que en las redes en las que se da una centralización de la intermediación del $100 \%$ tienen forma de estrella, y un único actor es el que conecta al resto de miembros de la red, mientras que una red que muestra una centralización de la intermediación de valor 0 tendrá una estructura circular en la que ningún actor tiene más capacidad que otro para interconectar a cualquier miembro de la red. 
de implicación comunitaria. Centrándonos en el primer tipo, en el cuadro 5 presentamos los estadísticos descriptivos de las medidas de cohesión utilizadas para analizar la estructura de las redes personales.

Cuadro 5. Estadísticos descriptivos de los indicadores de cohesión

\begin{tabular}{lcccc}
\hline & \multicolumn{2}{c}{$\begin{array}{c}\text { Desplazados } \\
(n=29)\end{array}$} & \multicolumn{2}{c}{$\begin{array}{c}\text { No Desplazados } \\
(n=31)^{a}\end{array}$} \\
\cline { 2 - 5 } Indicador & Media & $D T$ & Media & $D T$ \\
\hline Densidadb & .61 & .13 & .63 & .18 \\
Centralización de la intermediación & 10.09 & 9.08 & 7.9 & 9.4 \\
$N^{\circ}$ de cliques & 40.03 & 38.12 & 45.3 & 67.04 \\
\hline
\end{tabular}

aExiste un valor perdido en la población no desplazada. ${ }^{\text {b }}$ Debemos anotar que tanto la densidad como la centralización de la intermediación se expresan como porcentaje, de tal forma que una densidad de 0.61 equivale al $61 \%$ de las relaciones que se dan efectivamente en la red en comparación al total de vínculos posibles. *DT = Desviación tipo.

Fuente: Elaboración propia.

No se encontró diferencias estadísticamente significativas entre ambos grupos, no obstante, en resultados que se presentan más adelante se comentan algunos hallazgos significativos que matizan este hecho. Se observó que la densidad relacional en ambos colectivos es moderadamente elevada: superior al $60 \%$ en ambos grupos. La literatura sobre apoyo social sugiere que es necesario que la red personal cuente con un nivel suficiente de densidad que permita a ego recibir los recursos e información necesarios para garantizar un nivel óptimo de bienestar.

Sin embargo, en la literatura sobre capital social a nivel individual, en particular las aportaciones de Ron Burt (1992), demuestran que las redes excesivamente cohesivas dificultan el acceso a nuevas fuentes de información y recursos, puesto que este tipo de estructuras suelen ser altamente transitivas y el contenido de la información que fluye a través de la relación tienden a ser redundante en estos casos (Borgatti, Jones \& Everett, 1998; Burt, 1992; Ramos-Vidal, 2014, 2015). Por otro lado, la centralización de la intermediación muestra un valor moderado, inferior en ambos grupos al $10 \%$, si bien el valor de este indicador es ligeramente inferior entre la población local. En cuanto al número de cliques, se encontró en los dos casos que era elevado y superior en la población local. Esto parece mostrar mayor diversidad interna en la composición de la red.

Aunque el objetivo de este artículo no es mostrar las diferencias estructurales en las redes de hombres y mujeres, se ha identificado una serie de aspectos que merecen la pena ser rescatados por sus implicaciones para comprender el proceso de adaptación. La estructura de las redes personales de hombres y mujeres 
es significativamente diferente. Sin necesidad de distinguir entre desplazados y no desplazados, en la muestra completa las mujeres presentan un contexto relacional más cohesivo, en el que los vínculos son más densos respecto de los varones (densidad media de las redes de las mujeres es de $32.9 \%$ y $16 \%$ en el caso de los hombres). Estas diferencias son estadísticamente significativas (prueba chi cuadrado $5.654 \mathrm{p}<.05$ ).

Otro indicador que arroja diferencias notables entre hombres y mujeres es el nivel de centralización de la intermediación. En esta medida los valores de los hombres son significativamente superiores: la media de las redes de los varones es de $47.8 \%$ mientras que la de las mujeres es de $28.8 \%$ (prueba chi cuadrado $7.130 \mathrm{p}<.01)$. Esto refleja que los varones están en contacto con grupos más diversos y que existe mayor diversidad compositiva. Esta característica facilita el establecimiento de vínculos con grupos externos que, según diversos estudios, son los que permiten encontrar un nuevo empleo o ascender socialmente. Por el contrario, las mujeres disponen de un entorno relacional más cerrado en el que los vínculos son redundantes y dificultan el acceso a nuevas fuentes de apoyo. Para comprender lo mencionado, en los gráficos 1 y 2 se presentan las redes personales de una mujer y un varón en situación de desplazamiento.

Gráfico 1. Red personal de una mujer que lleva 9 años desplazada (caso 37)

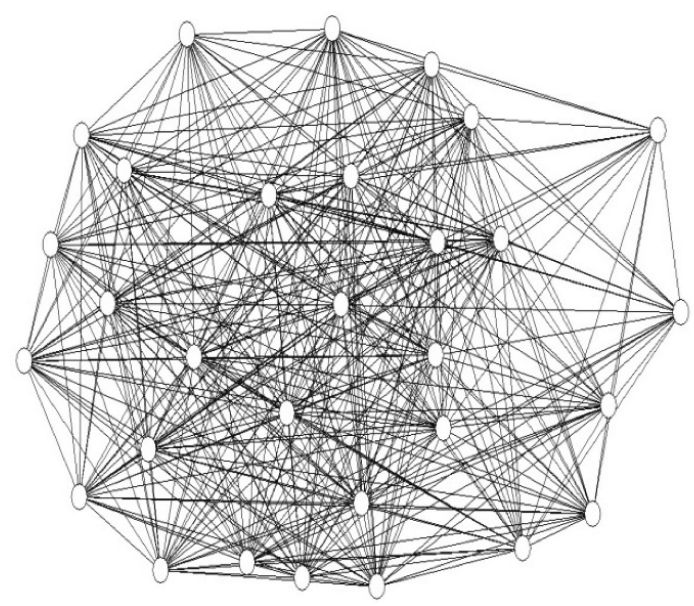

Fuente: Elaboración propia. 


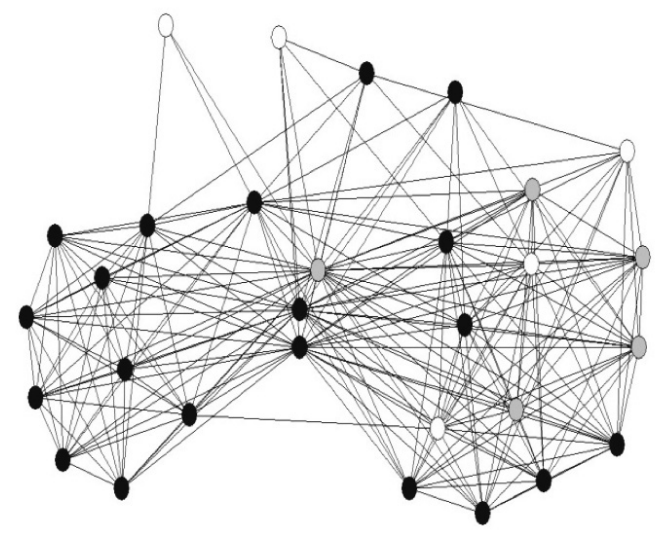

Fuente: Elaboración propia.

La visualización de las redes ofrece información interesante. El color de los nodos indica la procedencia de los actores (blanco = Barranquilla; gris = otra ciudad del departamento del Atlántico; negro = otra ciudad de Colombia). Atendiendo a la estructura se puede apreciar que la red de la mujer es más cohesiva que la del varón y solo se adivina un macrocomponente principal conformado por personas de Barranquilla. A priori se podría decir que la mayor presencia de alters de la comunidad de destino es un indicador de adaptación. Pero se requiere destacar que los estudios que analizan el proceso de adaptación de inmigrantes y refugiados en el contexto de destino muestran que las personas que reconstruyen su red únicamente con individuos del contexto de recepción, puede constituir un síntoma de la adopción de estrategias de adaptación cultural de tipo asimilativo, que a largo plazo derivan en problemas psicosociales (Berry, 1986, 1997; Ramos-Vidal, Holgado \& Maya-Jariego, 2014).

La red del varón muestra un contexto relacional muy distinto. La red está más fragmentada y es posible diferenciar dos grandes agrupaciones, una conformada por actores residentes en otra ciudad de Colombia (externa al departamento del Atlántico), y otra más heterogénea compuesta por residentes en Barranquilla, por habitantes de otra ciudad del Atlántico y por miembros de una ciudad ajena a esta región del país. El hombre, a pesar de llevar incluso más tiempo de desplazamiento que la mujer, presenta una diversidad relacional mayor en función de la procedencia de los alter. Este hecho refleja un proceso de adaptación híbrido en el cual la persona conserva sus contactos con el contexto de origen y al mismo tiempo incorpora progresivamente en su red a otros actores del con- 
texto de destino. Sin embargo, esta configuración no necesariamente puede ser la más satisfactoria en términos de adaptación. Transcurridos diez años desde el desplazamiento cabría esperar mayor presencia de actores del contexto de destino, por lo que es probable que la persona no cuente con suficientes fuentes de apoyo en la zona de reasentamiento.

También se observan variaciones de interés en las redes de las personas que trabajan en comparación con las que no desempeñan actividad remunerada. Las redes personales de los que trabajan son comparativamente menos cohesivas, su densidad media es del $23.7 \%$, mientras que la densidad media de los que no trabajan es de $34.6 \%$ (chi cuadrado $5.846 \mathrm{p}<.05$ ). Además, las redes de los entrevistados que trabajan presentan mayor centralización de la intermediación. La media de la centralización de la intermediación de los trabajadores es de $37.4 \%$, en comparación con el $24.5 \%$ de los que no trabajan (chi cuadrado $8.097 \mathrm{p}<.01$ ). Este dato es ilustrativo porque muestra desde un prisma estructural la función de integración social que cumple el desarrollo de actividades laborales. En el trabajo doméstico es menos probable que la realización de la actividad permita establecer nuevos contactos puesto que la tarea sucede en el domicilio (propio o ajeno). No obstante, realizar actividades por cuenta ajena o en el sector servicios implica a menudo tener que salir de la comunidad para trabajar y eso puede facilitar la construcción de nuevos contactos. Sin embargo, las personas que trabajan en el mismo domicilio y en la misma comunidad concentran sus relaciones — personales y profesionales - en un entorno acotado, aspecto que dificulta la ampliación de contactos con personas externas al núcleo duro de relaciones. En consecuencia, contar con un trabajo remunerado que permita entablar nuevas relaciones puede ser un indicador de adaptación en tanto puede facilitar el establecimiento de vínculos con personas del contexto de destino. Abajo se desglosan los resultados de la evaluación del SC y de la participación comunitaria en la población desplazada y no desplazada

Cuadro 6. Evaluación de procesos comunitarios

\begin{tabular}{|c|c|c|c|c|}
\hline \multirow[b]{2}{*}{ Tipo de proceso } & \multicolumn{2}{|c|}{$\begin{array}{c}\text { Desplazados } \\
(n=29)\end{array}$} & \multicolumn{2}{|c|}{$\begin{array}{c}\text { No Desplazados } \\
(n=32)\end{array}$} \\
\hline & Media & $D T^{*}$ & Media & $D T$ \\
\hline$\Sigma^{\star \star}$ Sentido de comunidad (SC escala completa) & 66.06 & 11.5 & 67.2 & 12.7 \\
\hline ¿ SC Satisfacción de necesidades & 16.1 & 3.4 & 16.2 & 3.8 \\
\hline$\Sigma$ SC Pertenencia & 15.8 & 3.4 & 16.5 & 3.3 \\
\hline$\Sigma$ SC Influencia & 16.2 & 3.4 & 16.6 & 3.9 \\
\hline$\Sigma$ SC Conexión emocional compartida & 17.7 & 3.2 & 17.7 & 3.5 \\
\hline$\Sigma$ Participación comunitaria & 12.1 & 4.9 & 13.1 & 4.8 \\
\hline
\end{tabular}

${ }^{*} \mathrm{DT}=$ Desviación tipo; ${ }^{*} \Sigma=$ Sumatorio

Fuente: Elaboración propia. 
No se observaron diferencias significativas entre los dos colectivos. Pero el cuadro 7 muestra que existe un alto grado de conexión entre el sentido de pertenencia y la participación en la comunidad.

Cuadro 7. Correlaciones entre los procesos comunitarios evaluados

\begin{tabular}{|c|c|c|c|c|c|c|}
\hline Variables & 1 & 2 & 3 & 4 & 5 & 6 \\
\hline 1. $\Sigma$ Sentido de comunidad & -- & & & & & \\
\hline 2. $\Sigma$ Satisfacción de necesidades & $.89^{\star}$ & -- & & & & \\
\hline 3. $\Sigma$ Pertenencia & $.83^{*}$ & $.70^{*}$ & -- & & & \\
\hline 4. $\Sigma$ Influencia & $.82^{*}$ & $.67^{\star}$ & $.53^{\star}$ & -- & & \\
\hline 5. $\Sigma$ Conexión emocional & $.82^{\star}$ & $.65^{*}$ & $.57^{\star}$ & $.57^{\star}$ & -- & \\
\hline 6. $\Sigma$ Participación comunitaria & $.45^{*}$ & $.41^{\star}$ & $.42^{\star}$ & $.35^{*}$ & $.34^{*}$ & -- \\
\hline
\end{tabular}

Nota: * $p<.01$

Fuente: Elaboración propia.

Un dato relevante es la intensa relación entre todas las dimensiones del SC y el proceso de participación. Un resultado consonante con los antecedentes que señalan que son dos procesos que se refuerzan y construyen mutuamente (Chavis \& Wandersman, 1990; Ramos-Vidal \& Maya-Jariego, 2014).

La estabilidad en el empleo es una variable capaz de modular la percepción y la interacción con la comunidad de acogida. Los resultados muestran que los trabajadores precarios, es decir, aquellos que no están vinculados formalmente a una organización a través de contrato profesional o los que realizan actividad esporádicamente sin disponer de contrato de trabajo, se sienten menos partícipes de la comunidad y manifiestan un menor sentido de pertenencia. Los trabajadores precarios obtuvieron una puntuación media de 9.5 en relación a los trabajadores no precarios que alcanzaron 15.3 (chi cuadrado $3.830 \mathrm{p}<.05$ ). Este resultado muestra los efectos de la precariedad laboral en la relación que el individuo establece con la comunidad de destino.

Después de realizar varios análisis exploratorios, en esta investigación se descubrió que el tiempo de residencia es clave para comprender el proceso de adaptación en el nuevo contexto de asentamiento. Se ha diferenciado a los desplazados en función del tiempo de desplazamiento distinguiendo dos grupos, uno conformado por los recién llegados (los que llevan menos de cinco años de desplazamiento) y otro por los desplazados de larga trayectoria (los que llevan más de cinco años). Una vez hecha esta clasificación se llevaron a cabo pruebas no paramétricas y se hallaron diferencias significativas entre los desplazados de mayor y menor tiempo en cuanto al grado de participación en la comunidad (chi cuadrado $4.245 \mathrm{p}<.05$ ). Los que llevan más de cinco 
años de residencia en la comunidad muestran mayor tendencia a participar si se los compara con los recién llegados. Al desarrollar la prueba de Levene asumiendo igualdad de varianzas, se encontró nuevamente que los desplazados con mayor tiempo de residencia se sienten más identificados con la comunidad. Esta tendencia se mostró en todas las dimensiones del SC a nivel de significación $\mathrm{p}<.05$.

Finalmente, para caracterizar a los desplazados se realizó un análisis de clúster por el procedimiento de las k-medias. Este análisis permite identificar subgrupos cuyas características son previamente desconocidas, pero que están basadas en variables especificadas por el investigador (Kaufman \& Rousseeuw, 2008). En este estudio se utilizaron como variables de agrupación 1) el tiempo de residencia en la comunidad de destino, 2) el número de miembros de la unidad familiar y 3) el nivel de ingresos mensuales. En el cuadro 8 se exponen los centros finales de los conglomerados.

Cuadro 8. Centros finales de los clústeres identificados

\begin{tabular}{lcc}
\hline & $\begin{array}{c}\text { Conglomerado } 1 \\
(n=8)\end{array}$ & $\begin{array}{c}\text { Conglomerado } 2 \\
(n=21)\end{array}$ \\
\cline { 2 - 3 } Variables de agrupamiento & Centros finales & Centros finales \\
\hline Tiempo de residencia (años) & 10.38 & 5.81 \\
$\mathrm{~N}^{\circ}$ de familiares con los que convive & 10.13 & 4.67 \\
Nivel de ingresos mensual (US\$) & 460.14 & 203.54 \\
\hline Fuente: Elaboración propia. & &
\end{tabular}

Se detectaron así dos conglomerados que convergen en dos iteraciones. La primera agrupación se caracteriza por contar con miembros que llevan un largo periodo de asentamiento en la comunidad de destino, que cuentan con una familia extensa y que disponen de una renta comparativamente elevada. Mientras que el conglomerado más numeroso está formado por desplazados que llevan aproximadamente cinco ańos asentados en la comunidad de destino, que conviven con la familia nuclear y que disponen de una baja renta per cápita. Los datos reflejan que el tiempo de residencia es un factor de importancia para comprender el proceso de adaptación. Se puede apreciar que existen dos perfiles claramente diferenciados de desplazados: uno mayoritario que lleva poco tiempo de residencia, percibe una renta baja y está en proceso de reagrupación familiar; y un segundo grupo de menor tamaño cuyo nivel de ingresos es más elevado, que lleva un largo tiempo en situación de desplazamiento y que ha conseguido reagrupar a gran parte de los miembros de la familia. En el siguiente apartado se discuten las implicaciones de los resultados descritos. 


\section{Conclusiones}

El desplazamiento provoca efectos de largo alcance en múltiples niveles. A lo largo de este trabajo se ha comprobado que las personas desplazadas pueden ver afectada la estructura de sus redes personales y las fuentes de apoyo social disponible. Tal proceso de pérdida y alteración de fuentes de apoyo ha sido analizado a través de la estructura y composición de las redes personales y se ha visto que el desplazamiento implica que la persona debe buscar nuevas fuentes de apoyo en el contexto de destino.

Se ha observado que el desarrollo de actividades profesionales es un indicador fundamental para establecer nuevas relaciones con grupos externos y que son potenciales fuentes de información y apoyo que posibilitan que los desplazados asciendan en la sociedad. Este dato refleja la importancia de diseñar itinerarios profesionales que faciliten a la población desplazada normalizar su situación. Desde esta óptica, el empleo se convierte en una vía privilegiada para obtener recursos económicos y sociales. La literatura sobre capital social describe con detalle la importancia de tejer vínculos con grupos diversos como mecanismo que posibilita acceder a oportunidades profesionales y personales (Granovetter, 1973). Es necesario que las instituciones lleven a cabo programas de intervención que induzcan a la integración de la población desplazada en el mercado de trabajo local y permitan aprovechar las características de la mano de obra, conectando oferta y demanda en un marco de relaciones laborales que potencie tanto el empleo estable y de calidad por cuenta ajena, como el trabajo autónomo en condiciones dignas y equiparables al trabajo asalariado.

El tiempo de residencia es un factor relevante al evaluar y comprender la situación de la población desplazada. Cuando la persona se desplaza, rompe vínculos con el contexto de origen. Esta ruptura, o el mero debilitamiento de las relaciones, se produce en todas las esferas de la vida. Cuando se moviliza la familia entera, los menores de edad ven desaparecer sus relaciones con la institución escolar, con los compañeros de clase y con los amigos del vecindario. Este fenómeno se reproduce cuando la persona es adulta y tiene que abandonar su trabajo. Por tanto, cada miembro de la unidad familiar que se desplaza pierde el apoyo social de la familia que queda atrás y pierde los recursos sociales de los contextos relacionales específicos en los que desenvuelve (escuela, trabajo, universidad, etc.). Esto conduce a pensar en la importancia de los contactos que el individuo establece en la comunidad de destino como alternativa para mejorar la calidad de vida y reducir la probabilidad de quedar confinado en contextos de exclusión social.

El SC ayuda a comprender la interacción entre el individuo y la comunidad, mientras que la participación es un indicador relevante que refleja las acciones 
que la persona está dispuesta a realizar para mejorar la calidad de vida en el nuevo asentamiento. En consecuencia, ambos procesos señalan dinámicas de inclusión y adaptación en la comunidad. Los resultados muestran que las personas que llevan más tiempo residiendo en la comunidad de destino se sienten más identificados y son más proclives a participar que los recién llegados. A medida que la persona se adapta a la comunidad, conoce más gente, cuenta con una historia compartida que facilita la identificación con la comunidad y progresivamente también se produce la aceptación por parte de la población local. Es necesario promover iniciativas de carácter comunitario que faciliten que las personas sean partícipes del cambio comunitario, adaptando las estructuras de participación tradicional (asociaciones de vecinos, organizaciones comunitarias) para que sean más inclusivas, accesibles y atractivas para la población desplazada. La identificación con la comunidad es un proceso perceptivo que facilita que la persona se involucre en iniciativas participativas y que adquiera mayor control y autodeterminación, que a su vez repercuten positivamente en el bienestar individual y en la satisfacción con la vida (Chavis \& Wandersman, 1990; Kloos et al., 2011; Wandersman, 2009).

Finalmente, se debe mencionar que los gobiernos de todos los niveles deben poner en marcha iniciativas que promuevan la inclusión de la población desplazada. Las políticas públicas implementadas deben actuar en diferentes frentes de forma simultánea para ofrecer una atención integral. En el caso de los menores desplazados es necesario promover acciones que faciliten una rápida adaptación a la dinámica escolar. Las instituciones que proporcionan servicios sociosanitarios han de instaurar protocolos de actuación que detecten de forma temprana a las familias desplazadas en riesgo de exclusión social. Por su parte, las instituciones que coordinan las políticas locales de empleo deben facilitar la adopción de medidas de discriminación positiva que canalicen a los trabajadores desplazados hacia empleos estables, o bien, que faciliten el reciclaje profesional. Por último, los gobiernos nacionales de los países aquejados por el problema del desplazamiento forzado deben promulgar iniciativas legislativas destinadas a preservar y restablecer los derechos de las personas que tuvieron que abandonar sus tierras por motivos ajenos a ellas. Pero no solo es necesario refrendar un complejo corpus legislativo, es preciso que los poderes del Estado hagan cumplir la ley convirtiéndose en el principal garante de los derechos de la ciudadanía.

\section{Limitaciones}

La comprensión profunda del fenómeno del desplazamiento obliga a puntualizar en algunos elementos de esta investigación. La particularidad del contexto 
colombiano dificulta la extrapolación de las conclusiones a otros países sin una adaptación previa. Por un lado, el número de casos analizado no constituye una muestra representativa de la población desplazada. Por otro, somos conscientes de la existencia de otro tipo de variables además de las descritas en este artículo que pueden mediar la identificación con la comunidad, pero este punto no impide afirmar que el grado de implicación comunitaria es un indicador fiable del proceso de adaptación en el contexto de destino. En última instancia hay otros elementos de las políticas públicas y de carácter macrosocial que influyen en el nivel de exclusión social de la población evaluada y que no han sido abordados en este manuscrito.

\section{Referencias}

ACNUR (2009). Politica del ACNUR sobre la protección de los refugiados y las soluciones en zonas urbanas. Septiembre de 2009. Recuperado el 1 de julio de 2015, de http://www.refworld.org/ docid/4b1f634e2.html

Albo, A. \& Ordaz, J. L. (2011). La migración mexicana hacia los Estados Unidos: Una breve radiografia. BBva Research. Documento de trabajo 11/05.

Ascodas. (2004). Asociación Colombiana de asistencia social, violencia y desplazamiento: casos Meta y Putumayo. Santafé de Bogotá.

Barrera, M., Sandler, I. \& Ramsay, T. B. (1981). Preliminary Development of a Scale of Social Support: Studies on College Students. American Journal of Community Psychology, 9(4), 435-447.

Berry, J. W. (1997). Immigration, Acculturation, and Adaptation. Applied Psychology, 46(1), 5-34.

Berry, J. W. (1986). The Acculturation Process and Refugee Behavior. En Williams, C. I. \& Westemeyer, J. (Eds.). Refugee Mental Health in Resettlement Countries (pp. 25-37). Nueva York: Hemisphere.

Borgatti, S., Everett, M. G. \& Freeman, L. C. (2002). Ucinet for Windows: Software for Social Network Analysis. Harvard: Analytic Technologies.

Borgatti, S., Jones, P. C. \& Everett, M. G. (1998). Networks Measures of Social Capital. Connections, 21(2), 36-51.

Builes, G., Arias, G. M. \& Minayo, M. C. (2008). Las migraciones forzadas por la violencia: el caso de Colombia, Ciência \& Saúde Coletiva, 13, 1649-1660. 
Burt, R. S. (1992). Structural Holes. Cambridge, Harvard University Press.

Caicedo, E. (1997). Conflictos sociales del siglo XIX. Bogotá: EA Colombia/Plaza y Janés.

Chavis, D. M., Lee, K. S. \& Acosta, J. D. (2008). The Sense of Community Index (sci) Revised: The Reliability and Validity of the SCI-2. Paper presentado en la 2o. International Community Psychology Conference. Lisboa, Portugal.

Chavis, D. \& Wandersman, A. (1990). Sense of Community in the Urban Environment: a Catalyst for Participation and Community Development. American Journal of Community Psychology, 18, 83-116.

Cohen, S. \& Wills, T. A. (1985). Stress, Social Support, and the Buffering Hypothesis. Psychological Bulletin, 98(2), 310-357.

Cruz, G. (2008). Informalidad regional en Colombia evidencia y determinantes. Desarrollo y Sociedad, 61, 43-85.

Demant, E. (2013). 30 años de la Declaración de Cartagena sobre Refugiados. Avances y desafíos de la protección de refugiados en Latinoamérica. Agenda Internacional, 31, 131-140.

De Miguel, V. \& Tranmer, M. (2010). Personal Support Networks of Immigrants to Spain: A Multilevel Analysis. Social Networks, 32(4), 253-262.

DeVellis, R. (1991). Scale Development: Theory and Applications. Newbury Park: Sage.

Diener, E., Sandvik, E., Seidlitz, L. \& Diener, M. (1993). The Relationship Between Income and Subjective Well-being: Relative or Absolute? Social Indicators Research, 28(3), 195-223.

Estrada, F. (2010). Estrategia y geografía política del conflicto armado en el Valle del Cauca. Análisis Politico, 23(69), 35-57.

Fisas, V. (2010). El proceso de paz en Colombia. Quaderns de Construcció de Pau, 17, 1-17.

Flores, P. \& Crawford, L. (2010). Identidades sin espacios de memoria. El caso del área metropolitana de Barranquilla (Colombia). Investigación \& Desarrollo, 14(2).

Forero, E. (2003). El desplazamiento interno forzado en Colombia. Conflict and Peace in Colombia: Consequences and Perspectives for the Future. Washington: Kellogg Institute.

Galor, O. \& Zeira, J. (1993). Income Distribution and Macroeconomics. The Review of Economic Studies, 60(1), 35-52. 
Granovetter, M. (1973). The Strength of Weak Ties. American Journal of Sociology, 78(6), $1360-1380$.

Guterres, A. (2011). Nansen Conference on Climate Change and Displacement. Statement. Oslo: United Nations High Commissioner for Refugees.

Harary, F. (1969). Graph Theory. Nueva York: Addison-Wesley.

Hernández, E. (1999). El desplazamiento forzado y la oferta estatal para la atención de la población desplazada por la violencia en Colombia. Reflexión Política, 1(1), 1-11.

IDMC. (2011). Colombia: Property Restitution in Sight but Integration Still Distant. Génova: Internal Displacement Monitoring Center.

Kaufman, L. \& Rousseeuw, P. (2008). Introduction, in Finding Groups in Data: An Introduction to Cluster Analysis. Hoboken: John Wiley \& Sons, Inc.

Kloos, B., Hill, J., Dalton, J. H., Elias, M. J., Wandersman, A. \& Thomas, E. (2011). Community Psychology: Linking Individuals and Communities. Wadsworth: Publishing Company.

Lleras-Muney, A. (2005). The Relationship Between Education and Adult Mortality in the United States. The Review of Economic Studies, 72(1), 189-221.

Lubbers, M., Molina, J. L., Lerner, J., Brandes, U., Ávila, J. \& McCarty, C. (2010). Longitudinal Analysis of Personal Networks: The Case of Argentinean Migrants in Spain. Social Networks, 32(1), 91-104.

Luce, D. \& Perry, A. (1949). A Method of Matrix Analysis of Group Structure. Psychometrika, 14(2), 95-116.

Martínez, M., García, M. \& Maya, I. (2001). El efecto amortiguador del apoyo social sobre la depresión en un colectivo de inmigrantes. Psicothema, 13(4), 605-610.

McCarty, C. (2002). Structure in Personal Networks. Journal of Social Structure, 3(1).

McMillan, D. \& Chavis, D. (1986). Sense of Community: a Definition and Theory. Journal of Community Psychology, 14, 6-23.

Naranjo, G. (2004). Ciudades y desplazamiento forzado en Colombia. El reasentamiento de hecho y el derecho al restablecimiento en contextos conflictivos de urbanización. En Albarracin, M. N. (Ed.). Desplazamiento forzado. Dinámicas de guerra, exclusión y desarraigo (pp. 279-309). Bogotá: UNAL. 
Novick, S. (Comp.). (2008). Las migraciones en América Latina: Políticas, culturas y estrategias. Buenos Aires: Clacso.

Ortega, D. (1988). Combatiendo por la paz. La Habana: Editora Política.

Oslender, U. (2007). Violence in Development: the Logic of Forced Displacement on Colombia's Pacific Coast. Development in Practice, 17(6), 752-764.

Palacio, J. \& Sabatier, C. (2002). Impacto psicológico de la violencia politica en Colombia. Barranquilla: Ediciones Uninorte.

Portes, A. (1995). En torno a la informalidad: ensayos sobre teoría y medición de la economía no regulada. México: Flacso México.

Ramos-Vidal, I. (2015). Aportaciones del análisis de redes sociales para evaluar el capital social a nivel individual y colectivo. En Contreras, C. \& Charry, C. I. (Coords.). Capital social: enfoques alternativos (pp. 57-81). México: Anthropos.

Ramos-Vidal, I. (2014). Influencia de la estructura de las redes personales sobre el desarrollo de procesos comunitarios en desplazados colombianos. Psychologia: Avances de la Disciplina, $8(1), 43-54$.

Ramos-Vidal, I., Holgado, D. \& Maya-Jariego, I. (2014). Las redes personales de los desplazados internos por la violencia política en Colombia, TRACE: Travaux et recherches dans les Amériques du Centre, 65, 51-68.

Ramos-Vidal, I. \& Maya-Jariego, I. (2014). Sentido de comunidad, empoderamiento psicológico y participación ciudadana en trabajadores de organizaciones culturales. Psychosocial Intervention, 23(3), 169-176.

Ramos-Vidal, I., Rodríguez, A., Palacio, J. \& Maya-Jariego, I. (2013). Redes personales de los menores trabajadores en familias desplazadas por la violencia politica en Colombia: indicadores para la evaluación de las acciones de cooperación internacional para el desarrollo. Informe final de resultados. Sevilla: Oficina de Cooperación al Desarrollo-Universidad de Sevilla.

Restrepo, J., Spagat, M. \& Vargas, J. F. (2003). The Dynamics of the Colombian Civil Conflict: A New Data Set. Centre for Economic Policy Research. Discussion Paper Series 4108. Recuperado el 11 de junio de 2017, de http://www.cepr.org/pubs/dps/DP4108.asp

Rubio, L. (2014) Desplazamiento inducido por violencia: Una experiencia global, una realidad mexicana. México: Concejo Gráfico. 
Sánchez, L. A. (2008). El desplazamiento forzado intraurbano: negación del derecho a la ciudad. En Riaño, P. \& Villa, M. (Eds.). Poniendo tierra de por medio: migraciones de colombianos en Colombia, Ecuador y Canadá (pp. 173-215). Medellín: Corporación Región.

Speer, P. W. \& Peterson, N. A. (2000). Psychometric Properties of an Empowerment Scale: Testing Cognitive, Emotional, and Behavioral Domains. Social Work Research, 24(2), 109-118.

Taylor, S. \& Brown, J. D. (1988). Illusion and Well-being: A Social Psychological Perspective on Mental Health. Psychological Bulletin, 103(2), 193-210.

Wandersman, A. (2009). Four Keys to Success (Theory, Implementation, Evaluation, and Resource/system Support): High Hopes and Challenges in Participation. American Journal of Community Psychology, 43(1-2), 3-21.

Recibido el 18 de agosto de 2015. Aceptado el 20 de abril de 2017. 